\title{
The effect of transient density profile shaping on transport in large stellarators and heliotrons
}

A. Dinklage ${ }^{1}$, R. Sakamoto ${ }^{2}$, M. Yokoyama ${ }^{2}$, K. Ida $^{2}$, J. Baldzuhn ${ }^{1}$, C.D. Beidler ${ }^{1}$, S. Cats ${ }^{3}$, K. Mc Carthy ${ }^{4}$, J. Geiger ${ }^{1}$, M. Kobayashi' ${ }^{2}$, H. Maaßberg ${ }^{1}$, S. Morita ${ }^{2}$, G. Motojima ${ }^{2}$,

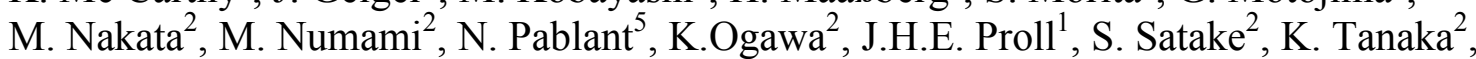

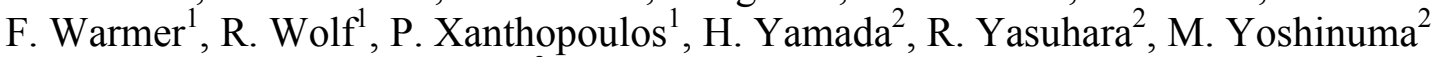
and the LHD Experiment Team ${ }^{2}$

${ }^{1}$ Max-Planck-Institut für Plasmaphysik, Greifswald, Germany

${ }^{2}$ National Institute for Fusion Science, Toki, Japan

${ }^{3}$ Eindhoven University of Technology, Eindhoven, The Netherlands

${ }^{4}$ CIEMAT, Madrid, Spain

${ }^{5}$ Princeton Plasma Physics Laboratory, Princeton NJ, USA

\section{E-mail contact of main author: dinklage@ipp.mpg.de}

\begin{abstract}
Transport studies of pellet fuelling experiments on LHD are reported. Spatio-temporal evolutions after pellet injection into LHD discharges show cases with central density increase on the time scale of particle transport processes. Both the temperature gradient and the density gradient change during the density relaxation, the latter even in sign. The resulting thermodynamic forces influence radial electric fields - both as a driving term but also by, e.g., affecting the $\mathrm{E}_{\mathrm{r}}$ dependence of ion transport. Magnetic fluctuations have been found to be induced by pellet injection but to die out with the relaxation of the pressure profile.
\end{abstract}

\section{Aspects of reactor scenario development in large stellarators and heliotrons}

Helical devices, namely stellarators [1] and heliotrons [2], are the main alternative magnetic confinement concept to the tokamak. Since the rotational transform in helical devices is predominantly generated by external coils, neither does a principal limitation of pulse lengths exist nor are strong toroidal plasma currents required. The latter prevents helical devices from being affected by current-driven instabilities and disruptions. Furthermore, the externally applied helical field results in more quiescent plasma behavior near operational boundaries, e.g. with regard to the plasma beta [3].

For fast plasma terminating events like thermal quenches, the confining field remains intact even in cases the plasma decays. Greenwald-density limits do not exist in helical devices but radiative limits depending on $B$ exist at even higher densities. Nonetheless, helical devices can operate at higher densities than tokamaks which is expected to ease divertor operation. At the same time, higher density at given Q leads to lower alpha-particle pressure resulting in a reduced drive of fast-ion driven instabilities.

An advantage with impact on reactor economy results from the fact that current-drive is, at most, a tool for minor adjustments of the magnetic configuration only. Therefore, the recirculating power in a potential helical fusion power plant is comparably small [4] .

While the advantages of helical devices result from the external generation of the magnetic geometry, the resulting 3D magnetic field also leads to issues. Consequences lie in 3D engineering and construction [5]. Even potential show-stoppers for helical devices results from trapped particles in local magnetic mirrors. These particles are susceptible to large, socalled neoclassical drift losses. The losses affect both the thermal plasma and collisionless 
fast-particle confinement. 3D transport shows a lack of neoclassical temperature screening [6] [7] (as in tokamaks). Therefore, helical-device-specific concepts to avoid impurity accumulation need to be further assessed (like the W7-AS HDH-mode [8] or the LHD impurity hole. The specific 3D magnetic field structure also affects the plasma rotation [9], turbulent transport [10][11] and is anticipated, e.g., to affect the occurrence of improved confinement modes.

Addressing potential show-stoppers has slowed the development of helical devices which are currently one technical generation behind tokamaks. Only the most recently built devices which produce sufficiently large plasma in the long-mean-free-path regime, namely LHD and W7-X, operate at parameters relevant to a potential helical fusion power plant. Increasing the size of helical devices, on the other hand, gives rise to new questions related to reliable fuelling schemes for reactor-grade plasmas.

Related to plasma fuelling, a specific issue in helical devices at fusion relevant temperatures is strong outward, neoclassical thermodiffusion contributing to particle fluxes. It has been shown that outward particle fluxes need to be compensated by core particle sources in order to avoid the depletion of particles in the plasma center [6]. Negative effects of lacking central particle sources are the loss of density control and loss of core heating capabilities due to cutoff in the plasma periphery, the decrease of fusion power and potentially the triggering of instabilities. Pellet injection is an option to provide particle sources [12] and this paper addresses to what degree transport effects fuel the plasma core. Therefore this study on LHD is highly relevant to the operation of Wendelstein 7-X for which ECRH provides a central energy source but no central particle refueling. At the same time, central fueling by NBI becomes the more ineffective the larger the density and the edge absorption becomes.

\section{Pellet fueling in the Large Helical Device}

Fig. 1 shows waveforms of an LHD discharge which was fueled with one hydrogen pellet consisting of $\mathrm{N}=1.5 \times 10^{21}$ particles. The target plasma had a central density of $\mathrm{n}_{\mathrm{e}}{ }^{0}=3 \times 10^{19}$ $\mathrm{m}^{-3}$. The total, mean port-through heating power was about $8 \mathrm{MW}$ coming from both perpendicularly injected NBI at $40 \mathrm{kV}\left(3.7 \mathrm{MW}, 4.6 \mathrm{MW}_{\text {peak }}\right)$ and tangentially injected NBI at $160 \mathrm{kV}(4.3 \mathrm{MW})$ acceleration voltage. The NBI at $40 \mathrm{kV}$ was modulated (20\%off $/ 80 \%$ on in

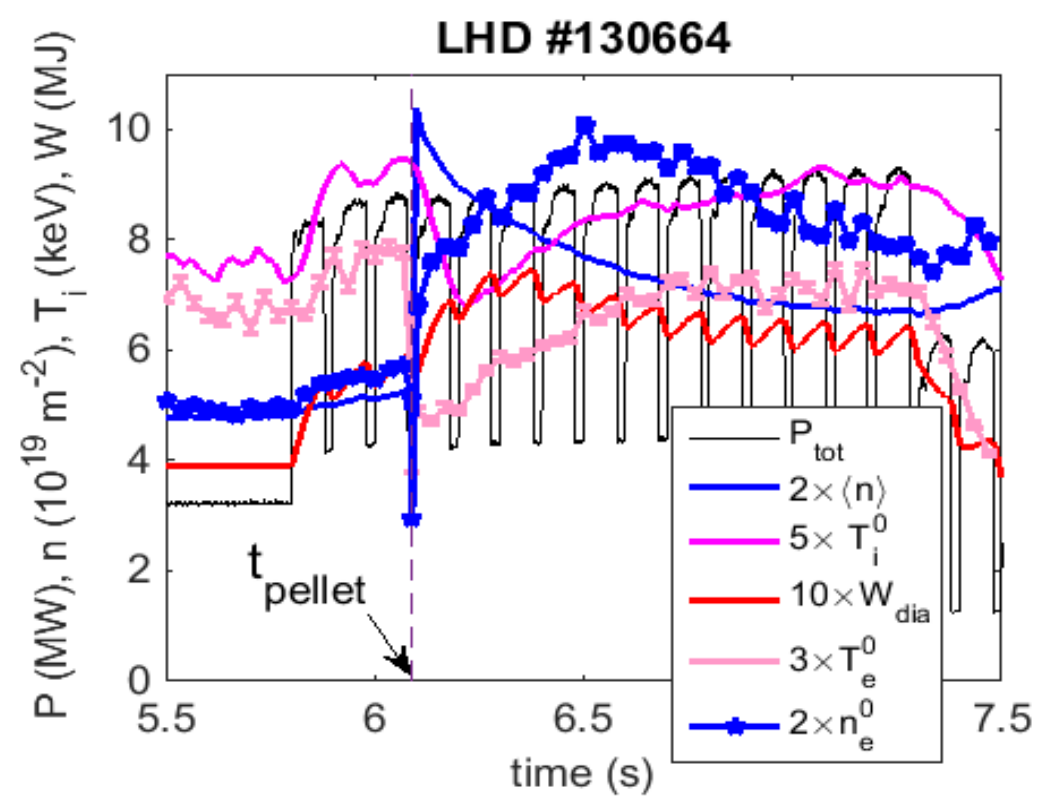

Fig. 1: Waveforms of heating power, mean density, central density, central ion- and electron temperature and diamagnetic energy in a pellet injection discharge. The pellet injection time is indicated at $\mathrm{t}=6.079 \mathrm{~s})$. The data are scaled. 
$100 \mathrm{~ms}$ ) to allow for background subtraction in charge-exchange recombi-nation measurements.

In response to the pellet injection at $t_{\text {pellet }}=6.079 \mathrm{~s}$, the mean density sharply increases (Fig. 1) and becomes stationary after about 1s. The central density shows responses on two time-scales. First, after the ablation phase, the central density is sharply increasing by about $20 \%$ on a time-scale of less than a few tens of milliseconds (Fig. 2). Second, the density is further increasing to about $70 \%$ above the value before pellet injection on a much longer time scale $(400 \mathrm{~ms})$ consistent with earlier observation [12]. On the same time scale, the temperatures recover. Later, after about $0.5 \mathrm{~s}$, the central density is decreasing again. The stored energy in the plasma shows a fast increase followed by a more moderate decrease consistent with the density and temperature waveforms. The radiated power was measured by bolometry to be about 1.5 MW (NBI on, 1.25 MW NBI off) and shows variations with the NBI modulation but remains almost unchanged after pellet injection in the NBI-on phases. To summarize these observations, it can be concluded that particle transport from peripheral pellet deposition is effective in fueling the plasma core in the analyzed LHD discharges on a time scale of the particle confinement time.

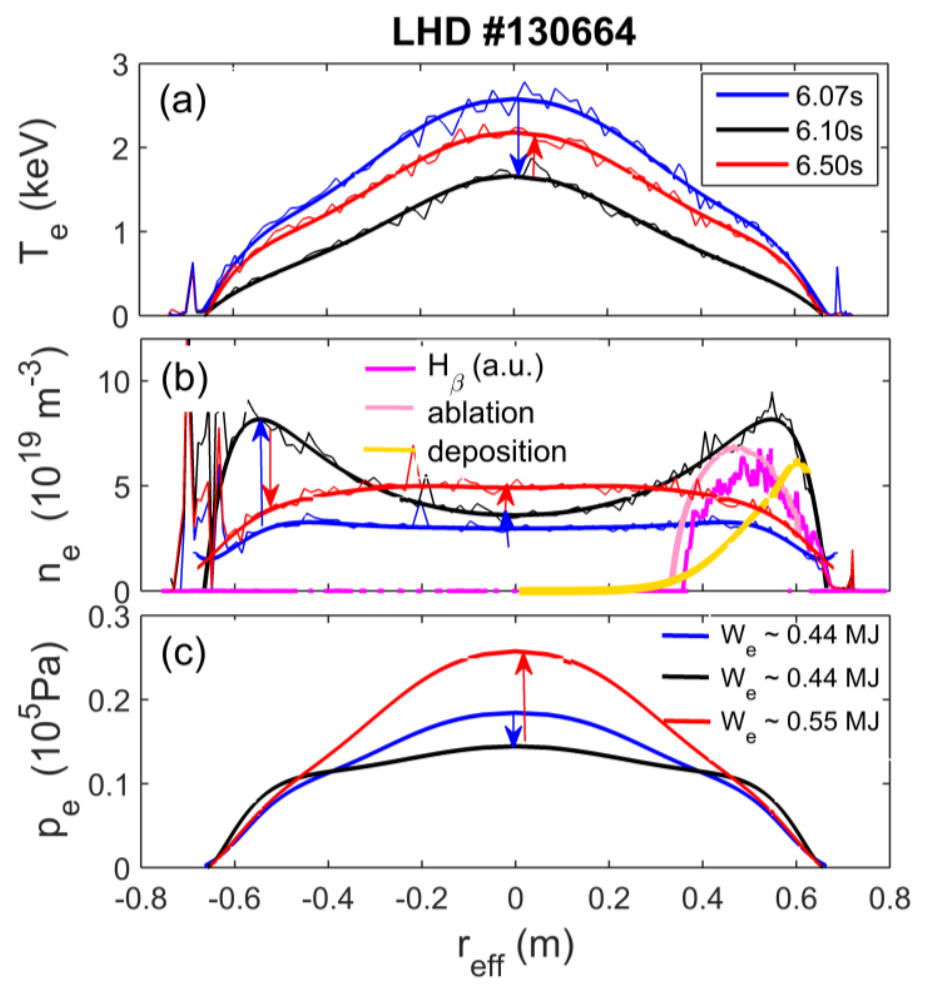

Fig. 2: Electron temperature (a), electron density and pellet ablation signals and deposition calculations (b) and electron pressure before pellet ablation and about $23 \mathrm{~ms}$ after pellet injection $(6.079 \mathrm{~s})$.

Electron temperature and -density profiles from Thomson-scattering before and after pellet injection are displayed in Fig. 2. Fig. 2(b) also shows $\mathrm{H}_{\beta}$ signals indicating neutral atom line emission from the pellet and ablation estimates from the neutral gas shielding model (NGS). Particle deposition calculations effects from the HPI2 code [13] including plasmoid drift are plotted as well.

The general trends in the electron profiles within the two phases of density increase are as follows: In the first phase (blue arrows), the injected particles are heated in an adiabatic process. The density profiles from Thomson scattering show an increase of $\mathrm{N}=0.98 \times 10^{21}$ 
particles in the plasma due to pellet injection, i.e. about $65 \%$ of the pellet atoms are brought within the last closed flux surface. The central density has increased by about $20 \%$. The electron pressure profile changes to become broader within the ablation phase. However, the HPI2 deposition calculations do not describe this increase of the central density. It is noted that the increase on a short timescale has been reported on LHD earlier [14]. Nonetheless, the peripheral peaks which contain the larger fraction of deposited particles and which result in strongly hollow density profiles match quite well with the deposition profiles. HPI2, however, predicts a very shallow deposition due to the grad-B outward drift acting on the pellet generated plasmoid.

In the second phase (red arrows), the central density increases further and the plasma is heated to recover partly in central temperature. The total energy is also increasing in line with observations of increased energy confinement with increasing density [15] (at about $\sim \mathrm{n}^{0.5}$ ). These findings confirm central fuelling to take place on a time scale $\left(\tau_{\mathrm{p}} \sim 400 \mathrm{~ms}\right)$ of a few times of the energy confinement time $\left(\tau_{\mathrm{E}} \sim 80 \mathrm{~ms}\right)$ assumed to be roughly consistent with the particle confinement time in the LHD plasmas investigated. More detailed investigations of particle transport are found in [15].

\section{Spatio-temporal evolution of transport relevant quantities after pellet injection}




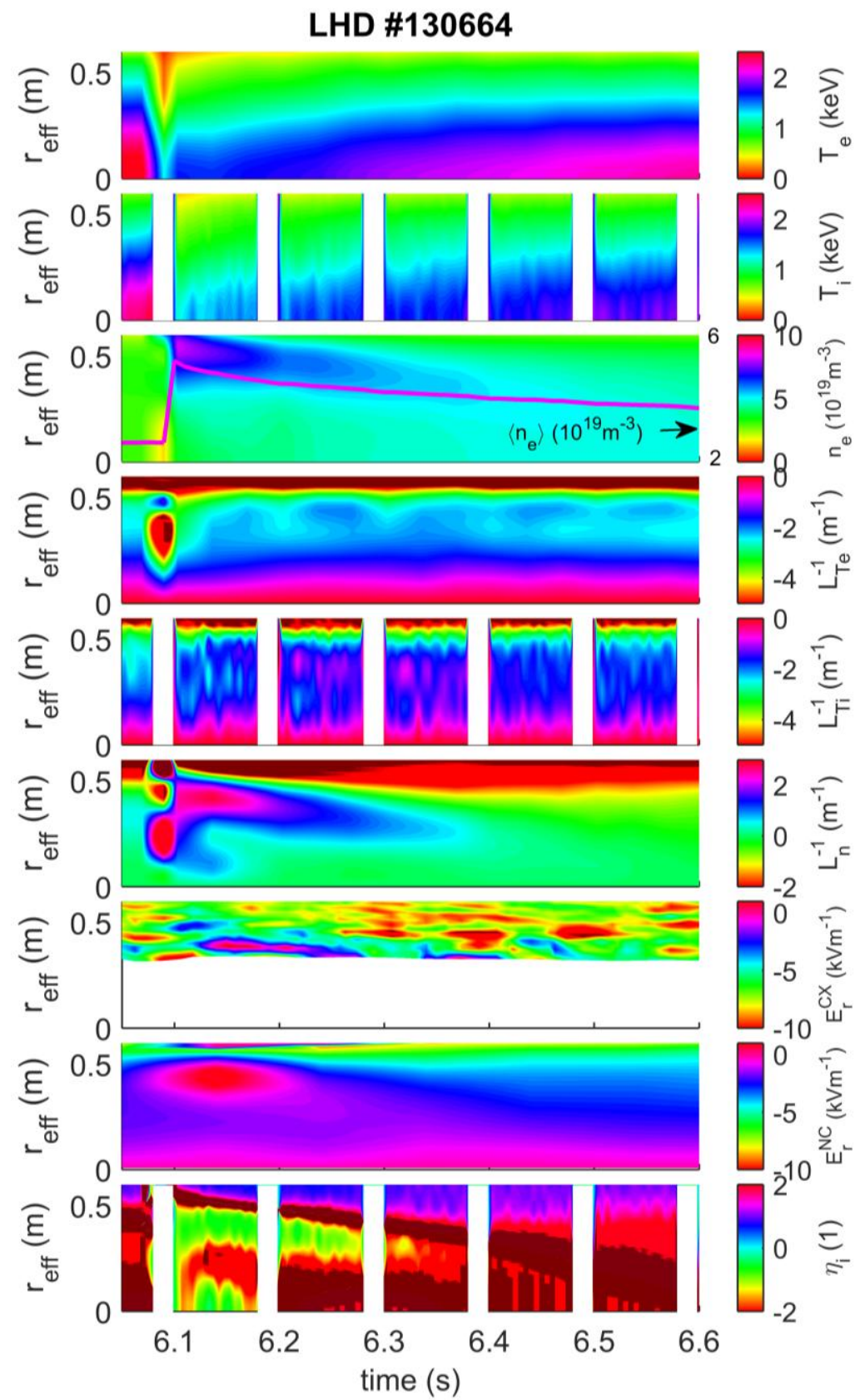

Fig. 3: Spatio-temporal evolution electron temperature $\left(T_{e}\right)$, ion temperature $T_{i}$, electron density $n_{e}$, respective gradient lengths $\left(\mathrm{L}^{-1}\right)$, measured radial electric field $\left(\mathrm{E}^{\mathrm{CX}} \mathrm{r}\right)$, the radial electric field from GSRAKE calculations $\left(\mathrm{E}_{\mathrm{r}}^{\mathrm{NC}}\right)$ and $\boldsymbol{\eta}_{\boldsymbol{i}}=\left(\boldsymbol{\nabla} \boldsymbol{T}_{\boldsymbol{i}} / \boldsymbol{T}_{\boldsymbol{i}}\right) /(\boldsymbol{\nabla} \boldsymbol{n} / \boldsymbol{n})$.

Fig. 3 summarizes the spatio-temporal evolution of transport relevant quantities in discharge 130664 of LHD. The space time plots document the transient filling up of a hollow density profile. The electron temperature and density was measured with Thomson scattering. The third plot also shows the mean density. The ion temperature and the radial electric field were measured by charge exchange re-combination spectroscopy. Periods without neutral beam injection have been covered with white patches. With fitted density and temperature profiles, the inverse gradient lengths $L^{-1}$ have been calculated and are displayed in the fourth to sixth plot from top. Both the electron temperature and ion temperature gradient lengths appear to significantly change only right after pellet injection $(\sim 30 \mathrm{~ms})$. In contrast, the density gradient 
length shows a significantly longer response time (like $\tau_{\mathrm{p}} \sim 400 \mathrm{~ms}$ ). The seventh plot shows of CXRS measurements of $E_{r}$ and the eighth plot shows radial electric fields determined (with GSRAKE [17][18]) from the ambipolarity condition applied to neoclassical ion and electron fluxes. Finally, the ratio of the inverse ion temperature gradient length to density gradient length is displayed. The figure clearly indicates negative $\eta_{i}<0$ in the region of large positive density gradients. Negative $\eta_{i}$ in the center are less relevant to transport since the gradients are small in the very center.

\section{Fluctuation response due to hollow density profiles}

Hollowness of the density profiles also leads to a local flattening with marginal, possibly slightly positive pressure gradients (cf. Fig. 4) in the discharges investigated. A criterion for the destabilization of resistive interchange modes is given by [18]

$$
p^{\prime} V^{\prime \prime}-\left\langle j_{\perp}^{2}\right\rangle-\left\langle j_{\|}^{2}\right\rangle<0
$$

which is fulfilled when a positive pressure gradient $p^{\prime}$ in regions of magnetic well $V^{\prime \prime}<0$ exceeds stabilization by Pfirsch-Schlüter $\left(\left\langle j_{\|}^{2}\right\rangle\right)$ and diamagnetic currents $\left(\left\langle j_{\perp}^{2}\right\rangle\right)$. It is stressed, however, that the magnetic configuration for the discharge investigated here is characterized by a global magnetic hill. Experimental findings are shown in Fig. 4 displaying the spatiotemporal evolution of the pressure gradient length (second plot from top) and waveforms of density, ion temperature and stored plasma energy.
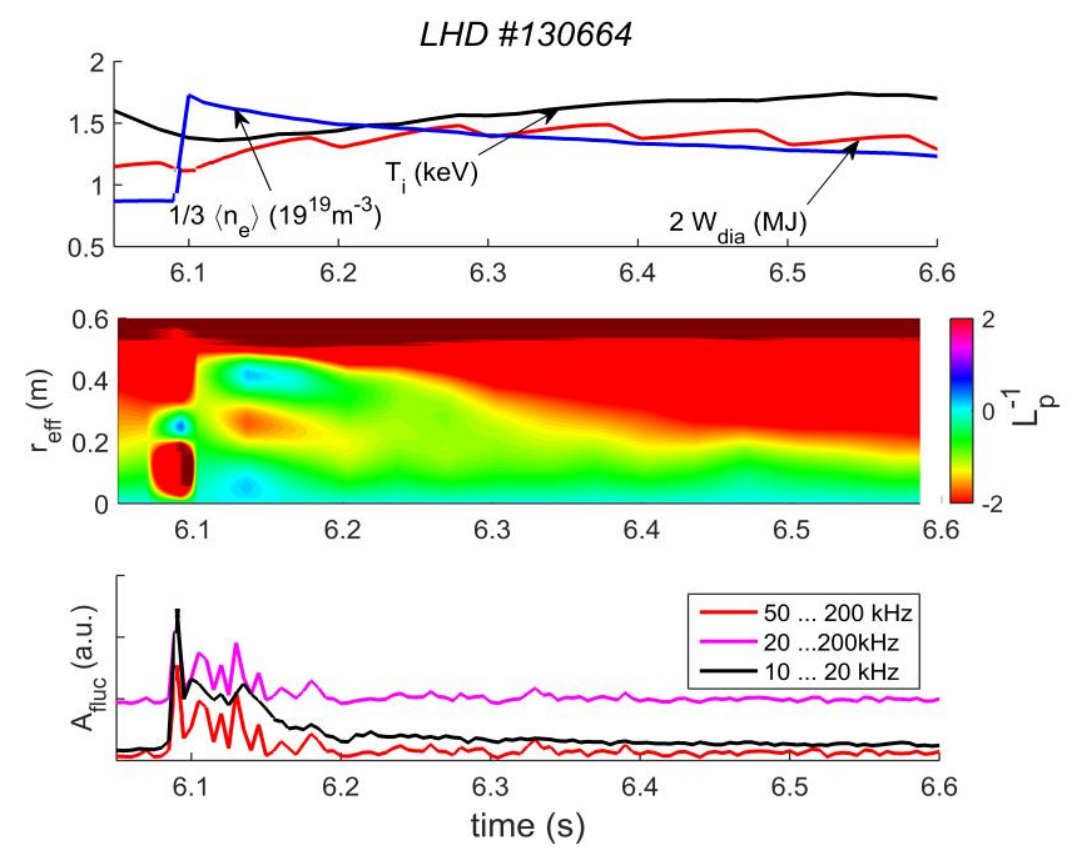

Fig. 4: Density, ion temperature and diamagnetic energy evolution (top plot) along with spatio-temporal evolution of inverse electron pressure gradient length (second plot from top). The third plot shows fluctuation levels in frequency ranges as indicated (from Mirnov data).

Furthermore, fluctuation levels in different frequency ranges from Mirnov coil data are shown in the bottom plot of Fig. 4. The fluctuation data are scaled and respective zero levels are shifted for the sake of visibility.

A more detailed analysis of all terms in the resistive interchange criterion, however, goes beyond the scope of this paper but it is reported as an experimental observation that hollow density profiles transiently lead to enhanced MHD fluctuation levels as evidenced by the 
comparison of the two bottom two figure. At the same time it is noted that neither density, nor temperature, nor energy signals shows a discontinuous evolution in these periods of redistributing pressure profiles. In other words, the transport relevant plasma quantities react mildly to unfavorable pressure profiles reflected by marginal or even slightly positive pressure gradient lengths as produced in the experiments analysed in this paper.

\section{Transport in transient responses after pellet injection}

The spatio-temporal measurements relevant to determine thermodynamic forces allow a comparison to local neoclassical theory. Neoclassical particle fluxes $\Gamma$ are not intrinsically ambipolar in helical devices and radial electric fields must arise to meet with the ambipolarity condition

$$
\Gamma_{e}=Z \Gamma_{i}
$$

In local neoclassical theory (e.g. [19]), the particle fluxes $\Gamma$ and energy fluxes $Q$ of species $\alpha$ are related to the inverse gradient lengths of densities $L_{n}^{-1}$ and temperatures $L_{T \alpha}^{-1}$ and $E_{r}$

$$
\begin{gathered}
\Gamma_{\alpha}=-n_{\alpha}\left[D_{11}^{\alpha}\left(L_{n_{\alpha}}^{-1}-\frac{q_{\alpha}}{T_{\alpha}} E_{r}\right)+D_{12}^{\alpha} L_{T_{\alpha}}^{-1}\right] \\
Q_{\alpha}=-n_{\alpha} T_{\alpha}\left[D_{21}^{\alpha}\left(L_{n_{\alpha}}^{-1}-\frac{q_{\alpha}}{T_{\alpha}} E_{r}\right)+D_{22}^{\alpha} L_{T_{\alpha}}^{-1}\right]
\end{gathered}
$$
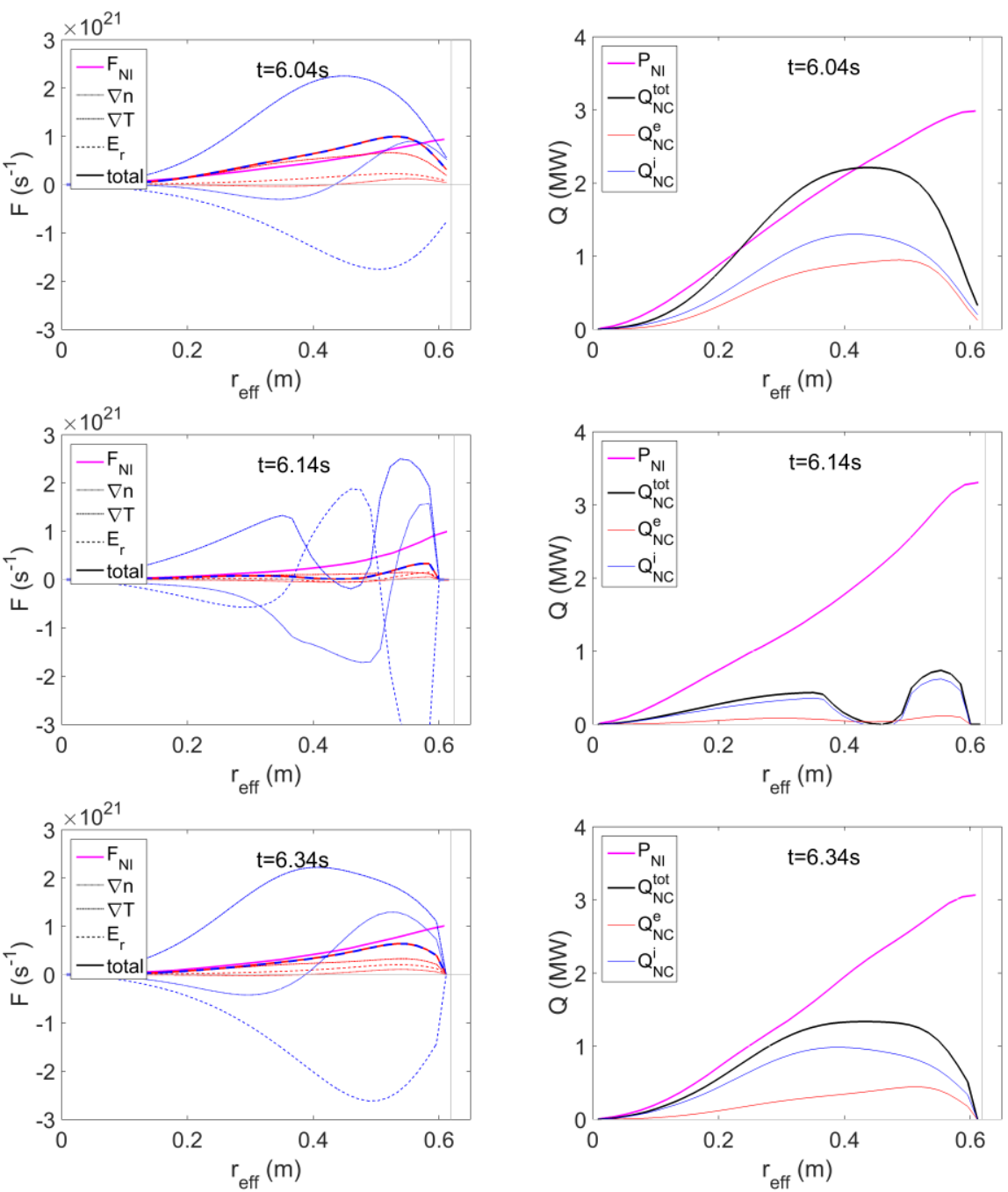

Fig. 5: Neoclassical particle (first column) and energy fluxes (second column) in comparison to power deposition calculations for $\mathrm{t}=6.04,6.14$ and $6.34 \mathrm{~s}$ in discharge LHD \#130664. Red lines refer to electrons. blue lines to ions. 
The transport co-efficients, depend on the radial electric field and the ambipolarity condition may lead to different solutions (roots) for $E_{r}$ [20][22]. For long-mean-free-path conditions at moderate or high densities, as for core plasmas discussed here, so-called ionroot conditions prevail, characterized by much larger ion transport coefficients $\left(D_{11}^{i} \gg D_{11}^{e}\right)$.

Fig. 5 shows a comparison of heat and particle source profiles from deposition calcula-tions with experi-mental profiles [18] due to neutral beam injection (magenta lines, recycling fluxes are not yet included in the sources). These source terms are compared to local neoclassical particle and energy fluxes $(q+F T)$ separated for ions and electrons for consecutive times before pellet injection and about 60 and $250 \mathrm{~ms}$ after pellet injection. The comparison shows fairly good agreement of these two independent calculations agreement in the plasma core for the times before and later after pellet injection (up to $r_{\text {eff }} / \mathbf{a} \sim 3 / 4$ for the particle fluxes and $r_{\text {eff }} / \mathbf{a}$ $\sim 1 / 2$ for the energy fluxes) between heating power and neoclassical fluxes. The neoclassical transport for these regions is clearly in the ion-root regime.

At about $60 \mathrm{~ms}$ after pellet injection, the local neoclassical description of the fluxes is much different over wide regions of the plasma. In regions of largest density gradients, the derived local neoclassical energy fluxes even vanish and it appears to be a fair conclusion that local neoclassical theory fails to reproduce the experimental findings for this case.

In order to discuss this discrepancy, the radial electric field is examined in more detail. With $\delta_{12}=D_{12} / D_{11}$ describing the impact of thermodiffusion vs. diffusivity, the radial electric field can be estimated to be

$$
\frac{Z e}{T_{i}} E_{r}=L_{n_{i}}^{-1}+\delta_{12}^{i} L_{T_{\alpha}}^{-1}
$$

Usually, the temperature and density gradients are negative (i.e. outward pointing) and thus $E_{r}<0$. Formally, however, the estimate for $E_{r}$ changes sign if

$$
L_{n_{i}}^{-1}>-\delta_{12}^{i} L_{T_{\alpha}}^{-1}
$$

and generates a positive radial electric field $E_{r}>0$. We call this hypothetical condition a positive ion-root. This situation is interesting to mitigate the influx of high-Z impurities. As a side-effect, the shape of the $E_{r}$ profile gives rise to sheared field and potentially negative values of the ratio of ion temperature gradient and density gradient, i.e. the dimensionless parameter relevant to the occurrence of turbulent fluxes [10][11].

Whether these conditions can be attained, however, depends on how much local, neoclassical theory is a valid description for cases $E_{r} \rightarrow 0$ since non-local effects may occur and transport coefficients diverge. Fig. 6 shows a comparison of radial electric fields calculated with local, neoclassical theory (solid lines) and charge-exchange recombination measurements [23]. The gray lines show $E_{r}$ before and about $260 \mathrm{~ms}$ after pellet injection. Both the calculated and measured $\left\langle E_{r}\right\rangle$ profiles are consistent with ion-root conditions. The measured ion-root reference is systematically smaller than the calculated mean $\left\langle E_{r}\right\rangle$.

Fig. 6 also shows clearly a transient response in $E_{r}$ measurements in regions with large density gradients at high densities. The resulting response is a decrease of $\left|E_{r}\right|$, i.e. the field becomes less negative when strong density gradients are generated by the pellet injection. 
The changes in the radial electric field are reproducible. Changes in the pellet fuelling and changes in the plasma parameters, however, lead to differences which will be reported elsewhere.

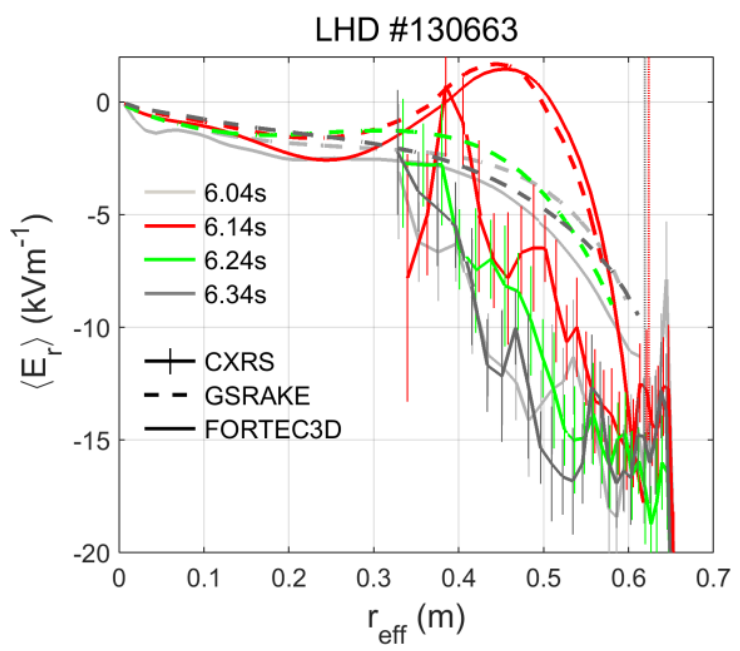

Fig. 6: Measurements of the radial electric field with charge exchange recombination spectroscopy (lines with error bars $\pm 25 \mathrm{~ms}$ averaging) and calculations of the radial electric field from temperature and density profiles. The pellets were injected at $t=6.079 \mathrm{~s}$, respectively. Parameters as for \#130664.

For the discharge analyzed here, the change in the radial electric field is most pronounced in the measurement about $60 \mathrm{~ms}$ after pellet injection, to arrive at very small negative radial electric fields. This time-slice is the one which shows largest density gradients and strongest hollowness, respectively. Corresponding local neoclassical calculations, however, even predict a change of sign (Fig. 6 solid red line) which was not confirmed by measurements.

It is anticipated that $E_{r} \rightarrow 0$ requires considering non-local radial coupling of particle fluxes. Neoclassical calculations with FORTEC 3D [24] retaining this coupling, however, show similar findings as local GSRAKE calculations (cf. Fig. 6).

\section{Summary}

Transient transport after pellet injection has been studied the Large Helical Device under long-mean-free-path conditions at moderate to high densities. Central fuelling has been observed in response to diffusive particle transport after pellet deposition in the periphery of the confinement volume. The initially (after pellet injection) very hollow density profile also results in marginally unstable pressure profiles and correlated fluctuations were observed. A response in the radial electric field was observed but not the change in sign as predicted from local neoclassical theory. The attained conditions represent an experimental case to further assess the role of non-local radial coupling in neoclassical transport and - at the same time to study the impact of the ratio of temperature and density gradients on turbulent mechanisms.

Acknowledgement: One of the authors (AD) would like to express his sincere gratitude to the LHD Experiment Team for conducting and supporting the reported study. The authors would like to thank the technical staff of the large helical device for their support of these experiments.

This work is partly supported by a Grant-in-Aid for Scientific Research of Japan Society for the Promotion of Science (JSPS) (No. 15H02336). This work is also partly supported by the 
National Institute for Fusion Science grant administrative budget (NIFS10ULHH021). This work has been carried out within the framework of the EUROfusion Consortium and has received funding from the Euratom research and training programme 2014-2018 under grant agreement No 633053. The views and opinions expressed herein do not necessarily reflect those of the European Commission.

\section{References}

[1] HELANDER, P., REP. PROG. PHYS. 77, 087001 (2014)

[2] WAKATANI, M., STELLARATOR AND HELIOTRON DEVICES (OXFORD UP, 1998)

[3] WELLER, A., et al., NUCL. FUSION 49, 065016 (2009)

[4] WARMER, F., et al., PLASMA PHYS. CONTR. FUSION 58, 74006 (2016)

[5] SCHAUER, F. et al., FUSION ENG. DESIGN 88, 1619 (2013)

[6] MAASSBERG, H. et al., PLASMA PHYS. CONTR. FUSION 41, 1135 (1999)

[7] VELASCO, J.-L., et al., PLASMA PHYS. CONTR. FUSION (2016) accepted

[8] McCORMICK, K. et al., PHYS. REV. LETT. 89, 015001 (2002)

[9] HELANDER, P., SIMAKOV, A.N., PHYS. REV. LETT. 101, 145003 (2008)

[10] NUNAMI, M., et al., PHYS. PLASMAS 19, 042504 (2012)

[11] PROLL, J.H.E., et al, PHYS. REV. LETT. 108, 245002 (2012)

[12] SAKAMOTO, R. et al., NUCL. FUSION 52, 083006 (2012)

[13] PÉGOURIÉ, B., PLASMA PHYS. CONTR. FUSION 49, R87 (2007)

[14] YASUHARA R., REV. SCI. INSTRUM. 85, 11 D822 (2014)

[15] TANAKA, K. et al., FUSION SCI . TECHNOL 58, 80 (2010)

[16] YAMADA, H. et al., NUCL. FUSION 45, 1684 (2005)

[17] BEIDLER, C. and D'HAESELEER, W., PLASMA PHYS. CONTR. FUSION 37, 463 (1995)

[18] YOKOYAMA M., et al., PLASMA FUSION RES. 7, 2403011 (2012)

[19] NÜHRENBERG, J., ZILLE, R. THEORY OF FUSION PLASMAS (VARENNA) 1987

[20] BEIDLER, C.D. et al., NUCL. FUSION 51, 076001 (2011)

[21] MYNICK, H. and HITCHON, W.N.G., NUCL. FUSION 23, 1023 (1983)

[22] YOKOYAMA M., et al., NUCL. FUSION 47, 1213 (2007)

[23] YOSHINUMA, M. et al., FUSION SCI . TECHNOL 58, 375 (2010)

[24] SATAKE, S. et al., J. PLASMA FUSION RES. 1, 002 (2006) 